\title{
Reaching Consensus and Highlighting Future Directions for Research: The Osteoarthritis Summit Breakout Sessions
}

\author{
Timothy Wright, PhD • Steven Goldring, MD
}

Received: 21 October 2011/Accepted: 17 November 2011/Published online: 3 February 2012

(C) The Author(s) 2012. This article is published with open access at Springerlink.com

Keywords osteoarthritis prevention · treatment diagnosis . classification

\section{Introduction}

The major goal of the Summit was to foster discussion among the participants in an effort to reach consensus on important topics in the prevention, diagnosis, and treatment of osteoarthritis (OA) and to highlight future directions for research. To that end, long breakout sessions were held at the end of both days of the summit. Here, we provide a summary of the sessions. To focus the discussion, participants were challenged to develop answers to questions posed by the co-chairs (addressed below). Much of the discussion was also focused around a proposal from John Hardin of the Arthritis Foundation to develop a concentrated, multidisciplinary, multi-institutional effort centered on the stabilization of cartilage matrix after joint trauma. Applying candidate pharmacologic agents and detecting the resulting stabilization with new imaging and molecular biomarkers could be a way to achieve concrete progress in preventing OA (see John Hardin's article elsewhere in this supplement).

\section{Classification and Pathophysiology}

Question 1: What criteria should be used to develop a classification system for OA?

A classification system is essential for all aspects of OA and needs to be applicable for detection and diagnosis, as well as for assessing the potential utility of an intervention. The current classification system for OA dichotomizes on primary ("natural") and secondary, but secondary is

T. Wright $\operatorname{PhD}(\bowtie) \cdot S$. Goldring MD

Hospital for Special Surgery,

535 East 70th Street,

New York, NY 10021, USA

e-mail: wrightt@hss.edu problematic because it could be argued that all OA is secondary to something, e.g., genetic factors and age. Perhaps a better way of dichotomizing OA is to employ the terms used for animal models of the disease: spontaneous or induced OA. The latter would include a specific event or identifiable intervening process, for example, a joint injury that led the patient to seek clinical evaluation.

Classification might also seek to combine symptoms (e.g., stiffness, pain, and functional limitations) with associated structural abnormalities in the same joint (as documented by physical examination or imaging). Structural or joint tissue compositional abnormalities also could be identified indirectly, prior to the stage at which structural changes can be detected by physical exam or imaging, perhaps through genotyping or the use of biomarkers detected in urine or blood samples.

The common imaging modalities and associated findings employed for diagnosis and classification of OA are well documented, including radiographic findings of joint space narrowing, osteophytes, and joint malalignment. Symptoms have been classified based on the presence and type of pain: aching, discomfort, stiffness, unease, troublesome, and "awareness" of the joint or on the severity of the symptoms - intermittent versus constant pain, fatigue, depression and anxiety, loss of sleep, and stiffness.

A classification system for OA could be refined by including the stage of the disease (e.g., preclinical molecular/metabolic, pre-radiographic, radiographic, and joint replacement stages). One could imagine a classification tree with a hierarchical approach. The recently proposed classification based on genetics, estrogen and menopausal status, and aging may be too general and nonspecific to be of use as a classification system. Similarly, classification systems based on the pathophysiological mechanism (e.g., biomechanical, injury, inflammation, and aging) or based on specific joint tissue involvement are premature at this time.

From the standpoint of clinical trials, all patients should be included regardless of classification. However, it was suggested that phenotypic classification of patients should 
be considered when selecting participants for clinical trials. For example, an intervention that is designed to target synovitis should be studied in patients with evidence of active synovial inflammation, since it is not always present throughout the clinical course of OA. Likewise, a therapy that is designed to target bone remodeling or cartilage repair optimally should be studied in patients with evidence of active involvement of these tissues.

Question 2: Should function and symptoms be included in the classification system?

We can gain insights from the utility of incorporation of functional and symptomatic criteria into classification systems for patients with rheumatoid arthritis (RA). For patients with $\mathrm{OA}$, functional assessments have been developed for clinical subsets of patients with specific sites of joint involvement. Functional assessments are usually self-reported, and self-reporting enables thorough and timely follow-up, but behavior modification should be assessed functionally by a trained observer when used as part of clinical trials. Differences between self-reports of function and objective measures of function have been reported and should be considered. Psychosocial patient characteristics affect selfreported functional outcomes more than they affect objective measures. Studies should include both standard self-reported measures, such as, WOMAC function, but also joint specific measures of function, such as the 6-min walk distance for knee OA.

Question 3: How do patient expectations and public health needs influence treatment selection and efficacy?

Meeting patient expectations with respect to the use of specific therapies must be balanced with evidence validated by an appropriate experimental assessment and testing (e.g., no arthroscopy for a degenerative meniscus without mechanical symptoms). Patient expectations center on a number of issues. For example, relief from pain and functional limitations are paramount, but concern about the future and the desire for the "least possible intervention" without making symptoms worse are important considerations as well.

Patients must be educated and informed regarding the structural and symptomatic changes that accompany the onset and progression of $\mathrm{OA}$ so that they have realistic expectations with respect to how these changes will affect their quality of life. With this information, they may more readily and realistically accept the known benefits of pharmacologic and nonpharmacologic interventions, such as exercise and weight loss, in preventing the disease.

Patient expectations should also be considered in the regulatory approach to demonstrate safety and efficacy of new OA treatments. Patients may well be less risk averse than investigators, institutional review boards, and the FDA when considering the value of new preventative, diagnostic, or treatment modalities for OA and the need to participate in clinical trials that would personally affect their disease.

From a public health standpoint, the need exists to emphasize the benefits of nonpharmacological therapies such as decreased obesity and increased activity and to develop a better understanding of the time course of initiation and progression of the disease. The impact of the doctor-patient relationship in OA should also be studied. Physicians typically do not have time to provide adequate lifestyle advice to the patient, nor does the physician have the tools to monitor adequately the impact of lifestyle changes on patient expectations and outcomes. Treatment approaches that integrate multidisciplinary health professionals rather than second and third opinions from other physicians would benefit patients with OA, especially in the absence of any current effect management algorithm for chronic OA.

Development of such sustainable, effective strategies should rely on a combination of biomechanical intervention, pharmacological agents, and weight loss with exercise programs with the goal of prevention or substantial delay in the need for joint replacement. Public health research should focus on the duration, safety, cost, and feasibility of such combined prevention and treatment approaches and consider behavioral economics, e.g., short-term incentives to lose weight as a means of accomplishing long-term sustainability.

Other public health issues surrounding OA include: reimbursement, reactive versus proactive therapy, presence of comorbid diseases, the need for an effective chronic disease management model, issues arising from OA patients repetitively cycling through the health care system without appropriate management of their disease, and the impact of new shifts in OA prevalence with prevalence rising dramatically in younger populations.

\section{Assessment}

Questions 4 and 5: What is the current status of tools to assess joint composition, structure, and function and how can they be used in diagnosis? and What are the optimal tools for diagnosis and for monitoring progression and response to therapy?

Imaging should be used to provide structural outcome(s) and in some studies may be used as a primary outcome along with pain and function. Recent advances in imaging techniques have been applied to the study and evaluation of patients with OA, and many of the most informative outcomes from imaging are just now coming to fruition in terms of use in clinical trials and consideration by the FDA for drug approval. Magnetic resonance imaging (MRI) allows evaluation of "cartilage health" using techniques that include delayed gadolinium-enhanced MRI of cartilage, so-called dGEMRIC, T2 mapping, and T1rho. These are widely available metrics and so are applicable for multi-site studies, though with dGEMRIC the risk imposed by double dose contrast and its use in patients with renal impairment at risk for the development of nephrogenic systemic sclerosis must be considered. Additional imaging modalities include optical coherence tomography, confocal microscopy, and Ramon spectroscopy, all of which are advancing to expand the global view of the joint in health and disease. MRI techniques and ultrasound have also been used to assess synovial pathology, and evidence exists that the presence of synovial inflammation is associated with less favorable clinical outcomes. Chemical exchange saturation 
transfer also shows promise as a valuable imaging tool, but is much less widely available.

Techniques for quantifying and assessing the structural and material properties of peri-articular bone have also been developed. Regional BMD is amenable to measurement, and additional techniques using X-ray, CT, and MRI are widely available. Mechanical assessments of tissues could include fluoroscopic imaging and dynamic MRI.

Except for PET imaging (which is limited by the expense), noninvasive tools for identifying and monitoring cell death have insufficient resolution to quantify cell death. Imaging cell death using Annexin V is a possibility, as are the use of DNA fragments and other available biomarkers (e.g., vimentin) [3]. Measurement of streaming potential in cartilage offers a precise and reproducible method of measuring proteoglycan content. However, the main limitation of this approach is the potential need for repeat arthroscopies.

Techniques for sensory assessment in patients with $\mathrm{OA}$ also are available and have been particularly informative in assessing the mechanisms of nociception in animal models of OA.

Considerable research in recent years has focused on the development of biomarkers that can be applied to gain insights into pathogenic mechanisms underlying OA; importantly, biomarkers can be utilized to assess the effects of therapeutic interventions. Biomarkers can be defined in general terms as objective indicators of normal biologic processes, pathogenic processes, or pharmacologic responses to therapeutic interventions and have the potential to decrease the length and cost of trials. Characteristically, biomarkers have been identified as molecules or molecular fragments that are released as a result of joint tissue metabolism, but additional markers are in development that are related to additional intra-articular processes such as inflammation. Discussions included review of the Osteoarthritis Biomarkers Network, which is a consortium of five sites, funded by the National Institutes of Health/National Institute of Arthritis, Musculoskeletal, and Skin Disease (NIH/NIAMS). Its goal is to develop and characterize new biomarkers and to refine existing OA biomarkers for clinical trials to gain insights into OA pathogenesis (A more detailed discussion and application of biomarkers is provided in the article by Linda Sandell in this supplement).

\section{Treatment}

Question 6: What specific pathophysiological processes responsible for tissue alterations and symptoms should be therapeutically targeted?

Processes that should be considered include synovitis, cartilage loss, bone remodeling (bone marrow lesions, periarticular bony changes), osteochondral repair response, muscle weakness, and abnormalities in the meniscus, ligaments and entheses with or without associated joint laxity. The ideal therapeutic needs to target pain and related symptoms in addition to joint structure and to be effective within a reasonable time. Importantly, OA is a "whole joint" disease that affects all of the tissue components of the joint. In individual patient subsets, a single tissue, e.g., cartilage or bone, may be the primary target of the pathologic process, but optimal therapies may need to target multiple tissues.

Question 7: What are the optimal therapeutic targets?

Organizing OA phenotypes into "syndromes" provides a potentially useful approach in constructing a rational framework for optimizing treatments. The so-called metabolic syndrome that affects cartilage homeostasis, bone remodeling, and inflammation would be such an example. Other potential organizing principles include mechanical and structural syndromes (e.g., malalignment, obesity, joint incongruity, and joint instability); age-related (including sarcopenia); and inflammatory (associated with synovitis).

Question 8: What interventions (medical and surgical) can be employed to prevent or slow the progression of OA and when should they be employed?

Given that many animal models of OA are joint injury models, human OA "syndromes" that are initiated by acute joint injury represent useful conditions for understanding the pathophysiological processes associated with $\mathrm{OA}$ and for evaluating the effect of therapeutic interventions. For example, certain acute joint injuries in humans are accompanied by an increase in aggrecan (fragment) release from articular cartilage. This process is dependent on the activity of "aggrecanses" that can be potentially targeted with inhibitors and the efficacy of the intervention assessed using presently available imaging techniques and/or blood and urine biomarkers. Collagen degradation that may lead to irreversible cartilage damage can also be observed shortly after acute joint injury, and therapies that target this pathologic process can be assessed. "Is there target engagement?" is a critical question that may well depend on the ability of an imaging outcome to show preserved health of the cartilage.

Prevention is generally ignored in the consideration of therapeutic intervention strategies. The comparative costeffectiveness of different strategies is important; effective interventions exist for many pain-related problems, such as, pain catastrophizing, but these are rarely used. Weight loss for obese knee OA patients is effective for pain (see the article by Stephen Messier in this supplement). Reimbursement is a major obstacle in implementing prevention measures. Will insurance companies pay for supervised exercise and weight loss intervention? Such an approach is not currently considered a "medical benefit," and so would be considered a special case by third party payers. NIH-funded randomized clinical trials of other nonpharmacological interventions are ongoing, so hopefully the necessary additional data will be forthcoming.

Many pharmacological agents that target synovial inflammation and cartilage and bone remodeling are available. In general, they are designed for systemic administration. In cancer therapy, liposomes and nanoparticle delivery systems are being used for targeting specific sites. Similar approaches for local intra-articular delivery of agents to affected joints are being developed for treatment of OA.

Surgical interventions are improving steadily, including the advent of distraction technology to temporarily unload the joint and allow a period of prolonged healing. The role of excessive joint loading in the onset and progression of OA has been extensively studied. Importantly, both osteotomies and joint 
distraction have been shown to modify joint mechanics and loading and to modify the course and rate of progression of OA. Surgical techniques to correct femoral acetabular impingement hold promise for improving the outcomes in patients with hip joint structural abnormalities, but it is unclear at this time which forms of malalignment are risk factors for $\mathrm{OA}$ and whether the surgical procedures will avert the development of OA.

\section{Outcome Measures and Clinical Assessments}

Question 9: What measures should be used as endpoints in clinical trials of patients with OA?

Demonstrating the efficacy of early intervention is critical to insure a major breakthrough in OA. However, given the state of the current art, it is more likely that patients would be enrolled who already have clinical and/or structural evidence of OA and for whom the intervention is designed to slow or prevent the OA progression. Effective endpoints would be pain and function that can be measured using a variety of assessment tools including Western Ontario and McMaster Universities Osteoarthritis Index: WOMAC; pain characterization (McGill short form); Intermittent and Constant Osteoarthritis Pain (ICOAP) questionnaire that measures pain comprehensively in the knee and hip; and Knee and Hip Injury and Osteoarthritis Outcome Scores. Good performance based measures include Timed Up and Go, timed walk (e.g., 6 min), stair climb and chair stands. Optimal measures of physical performance need to incorporate assessment of functional activities that are relevant to the patient's needs. An effective therapy would improve performance beyond just joint function.

To power a trial, it would be best to pick two primary endpoints, one symptom based and one structure based. To gain maximum information, the suggestion would be to use all the technology (and measures) that can possibly be incorporated; however, requiring too many patient-reported outcomes becomes a burden to study participants who are often elderly. In a drug development program, the number of outcomes is highly focused rather than broad as for an exploratory study. Question 10: Are different endpoints needed for specific clinical subsets?

Both floor and ceiling effects may apply for different patient subsets. In quality of life utilities, for example, the SF60 has a floor effect so it is not as discriminant in disease populations, while the EQ-5D has a ceiling effect so it is not as discriminant in healthy people. The NIH PROMISE study can help avoid ceiling/floor effects. An OA computer-adapted tool has been developed to avoid floor and ceiling effects [5]. The self-reported The Compendium of Physical Activity [1] has been translated into joint loads. Accelerometry can provide objective physical activity data and shows that self-reports exaggerate levels of activity [4]. Joint shape and how it impacts underlying tissue architecture is another emerging outcome [2].

Question 11: Who should define the priorities?

Although the obvious answer is the patients, the entities that pay the medical bills realistically define the priorities. However, based on current limitations in the FDA regulatory approach to OA treatments, candidate therapies cannot be effectively prioritized. Working on the basic biology of the disease will help advance this effort, as will the OARSI FDA OA initiative.

Acknowledgments Funding for the summit was supplied by: Amgen, Inc. (Thousand Oaks, CA); Endo Pharmaceuticals (Newark, DE); Genzyme Biosurgery (Cambridge, MA); Novartis Pharmaceuticals Corp. (East Hanover, NJ); Pfizer, Inc. (New York, NY); Zimmer, Inc. (Warsaw, IN). Partial support was also provided by the NIH-funded Weill Clinical Translational Science Center (NIH project no. 3UL1RR024996).

Disclosures Each author certifies that he or she has no commercial associations (e.g., consultancies, stock ownership, equity interest, patent/licensing arrangements, etc.) that might pose a conflict of interest in connection with the submitted article.

Open Access This article is distributed under the terms of the Creative Commons Attribution Noncommercial License which permits any noncommercial use, distribution, and reproduction in any medium, provided the original author(s) and source are credited.

\section{References}

1. Ainsworth BE, Haskell WL, Herrmann SD, Meckes N, Bassett DR Jr, Tudor-Locke C, Greer JL, Vezina J, Whitt-Glover MC, Leon AS. 2011 Compendium of Physical Activities: a second update of codes and MET values. Med Sci Sports Exerc. 2011;43:1575-81.

2. Baker-LePain JC, Lane NE. Relationship between joint shape and the development of osteoarthritis. Curr Opin Rheumatol. 2010;22:538-43.

3. Gobezie R, Kho A, Krastins B, Sarracino DA, Thornhill TS, Chase M, Millett PJ, Lee DM. High abundance synovial fluid proteome: distinct profiles in health and osteoarthritis. Arthritis Res Ther. 2007;9:R36.

4. Ham SA, Ainsworth BE. Disparities in data on Healthy People 2010 physical activity objectives collected by accelerometry and self-report. Am J Public Health. 2010;100 Suppl 1:S2638 .

5. Jette AM, McDonough CM, Ni P, Haley SM, Hambleton RK, Olarsch S, Hunter DJ, Kim YJ, Felson DT. A functional difficulty and functional pain instrument for hip and knee osteoarthritis. Arthritis Res Ther. 2009;11:R107. 